Binghamton University

The Open Repository @ Binghamton (The ORB)

Mechanical Engineering Faculty Scholarship

Mechanical Engineering

2019

\title{
A tunable Electrostatic MEMS Pressure Switch
}

Mark Pallay

Binghamton University--SUNY, mpallay1@binghamton.edu

Ronald N. Miles

Binghamton University--SUNY, rmiles@binghamton.edu

Shahrzad Towfighian

Binghamton University--SUNY, stowfigh@binghamton.edu

Follow this and additional works at: https://orb.binghamton.edu/mechanical_fac

Part of the Mechanical Engineering Commons

\section{Recommended Citation}

Pallay, M., Miles, R. N., \& Towfighian, S. (2019). A tunable Electrostatic MEMS Pressure Switch. IEEE TRANSACTIONS ON INDUSTRIAL ELECTRONICS. doi: 10.1109/TIE.2019.2956377

This Article is brought to you for free and open access by the Mechanical Engineering at The Open Repository @ Binghamton (The ORB). It has been accepted for inclusion in Mechanical Engineering Faculty Scholarship by an authorized administrator of The Open Repository @ Binghamton (The ORB). For more information, please contact ORB@binghamton.edu. 


\title{
A tunable Electrostatic MEMS Pressure Switch
}

\author{
Mark Pallay, Ronald N. Miles, and Shahrzad Towfighian,
}

\begin{abstract}
We demonstrate a tunable air pressure switch. The switch detects when the ambient pressure drops below a threshold value and automatically triggers without the need for any computational overhead to read the pressure or trigger the switch. The switch exploits the significant fluid interaction of a MEMS beam undergoing a large oscillation from electrostatic levitation to detect changes in ambient pressure. If the oscillation amplitude near the resonant frequency is above a threshold level, dynamic pullin is triggered and the switch is closed. The pressure at which the switch closes can be tuned by adjusting the voltage applied to the switch. The use of electrostatic levitation allows the device to be released from their pulledin position and reused many times without mechanical failure. A theoretical model is derived and validated with experimental data. It is experimentally demonstrated that the pressure switching mechanism is feasible.
\end{abstract}

Index Terms-Electrostatic Levitation, MEMS, Pressure Switch, Sensors

\section{INTRODUCTION}

A CCURATELY sensing ambient pressure is very important for the aerospace, automotive, manufacturing, and medical industries. Traditional pressure sensors detect pressure with a movable plate (diaphragm) that is suspended above an evacuated cavity. The ambient pressure pushes on the top surface of the diaphragm, and the deflection is measured and converted into an electrical signal [1]-[3]. In micro-electromechanical systems (MEMS), this is typically achieved using piezoelectric materials that generate a voltage when undergoing mechanical strain, or through piezoresistive strain gauges. MEMS pressure sensors have advantages over their larger counterparts because they can fit in much smaller spaces, provide accurate measurements of pressure at a single point, and have very cheap bulk fabrication costs.

One caveat of MEMS pressure sensors is they can not act as pressure switches. MEMS sensors continually give a quantitative measurement of the pressure, which is read by a computer or fed into a control circuit. However, some applications only need to trigger a response when the pressure passes a certain value, such as turning on a tire pressure light on the dashboard of a car. This requires the output of

Manuscript received August 19, 2019; revised November 17, 2019; accepted November 20, 2019. This work was supported in part by the National Science Foundation Division of Electrical, Communications, and Cyber Systems under Grant No. 1608692.

Mark Pallay, Ronald N. Miles, and Shahrzad Towfighian are with the Mechanical Engineering Department, Binghamton University, Binghamton, NY, USA, (e-mail: mpallay1@binghamton.edu, miles@binghamton.edu, stowfigh@binghamton.edu - corresponding author) . a MEMS pressure sensor to be continually monitored and interfaced with a switch that is triggered when the pressure passes the specified value [4]. A MEMS pressure switch would provide the advantages of MEMS technology without the need to constantly monitor the pressure level, which could reduce the manufacturing cost and greatly simplify the complexity of the entire system.

One way a MEMS pressure switch can be realized is to use bifurcations in nonlinear systems. Bifurcation sensors exploit sudden changes in beam dynamics to detect when the sensed quantity passes a threshold value. Many bifurcation sensors exploit hysteresis, which can be triggered when some parameter of the system changes from an outside influence. These sensors can be extremely sensitive and have high signalto-noise ratios because of the dramatic difference between their on and off states [5]. One of the most common types of bifurcation sensors is a mass sensor, which uses the shifting natural frequency from the added mass of the sensed particle to induce a dramatic jump in the sensor response when the particle count passes a threshold [6], [7]. Other types of bifurcations that have been used for sensing are bi-stability [8], and the pull-in instability [9], [10]. Pull-in bifurcation sensors are particularly useful for a MEMS pressure switch because their bifurcation is associated with two conductors coming into contact with each other, which can be seen as the closing of a switch. If the pull-in bifurcation could be controlled by the ambient pressure, then it could be used to create a MEMS pressure switch.

The actuator proposed by the authors in a previous study [11] can be used as a bifurcation pressure switch because the dynamic pull-in instability is dependent on the ambient pressure. The switch uses an electrostatic levitation electrode configuration [12]-[14] with two input voltages. A cross section of the electrode arrangement is shown in Figure 1. A cantilever is suspended above three electrodes fixed to an insulating layer on the substrate. The side electrodes (red) are given a high voltage relative to the center electrode (green) and beam (blue) to induce electrostatic levitation. The electric field from the side electrodes pulls on the top of the beam more than the bottom, producing a net force upward. A second voltage is placed on the center electrode to generate an attractive force that pulls the beam back down, which is similar to a parallelplate capacitor. The actuator can act as a switch by applying the pull-in voltage to the center electrode to initiate pull-in, creating a connection between the beam and center electrode. If a large voltage is applied to the side electrodes, the beam can be released from pull-in, opening the switch [15].

Electrostatic levitation has a number of interesting attributes that are very useful for pressure sensing. Most importantly, it 


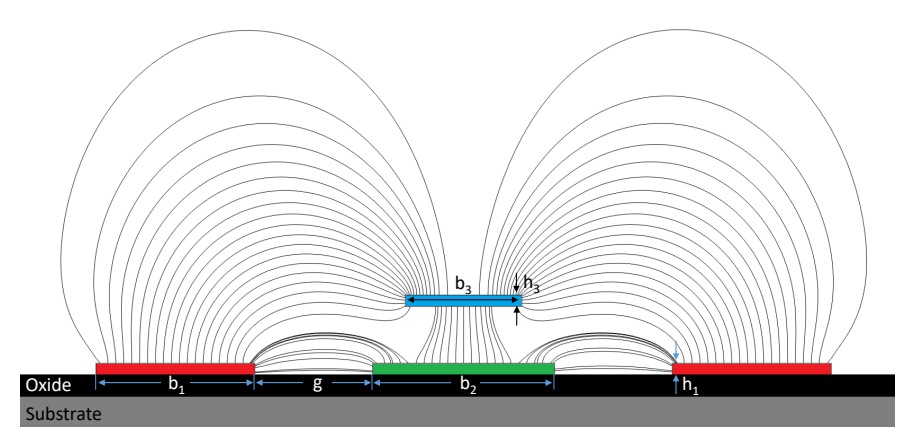

Fig. 1. Electrode layout of the threshold pressure switch with geometric parameters and electric field. The value of the geometric parameters are given in Table I.

can produce very large oscillations over an order of magnitude greater than the anchor height of the beam [16], [17]. Previous experimental results showed the beam oscillating almost 25 $\mu \mathrm{m}$ peak-to-peak, with the tip repeatedly tapping on the center electrode. As reported by the authors in [17], the beam dynamics are heavily influenced by the air spring effect when the dynamic amplitude is large. The large oscillation at a high frequency creates a compressible cushion of air between the beam and substrate and significantly limits the dynamic amplitude, which is also affected by squeeze film damping. The strong coupling between beam and fluid dynamics makes electrostatic levitation an ideal candidate for measuring ambient pressure. Another advantage of using electrostatic levitation for the pressure switch is the beam can be released from its pulled-in position as demonstrated in [15]. In traditional parallel-plate MEMS, pull-in often causes breakage and permanent failure, which makes electrostatic switches less reliable. However, in this design the switch can be reused many times without failure.

One drawback of electrostatic levitation is the high voltage requirement. The levitation force is inherently weak because it uses fringing electrostatic fields and requires voltages upwards of $100 \mathrm{~V}$ to achieve a large oscillation amplitude. Because of recent advances in ASIC designs, high DC voltages can be easily produced. In applications where high voltages are of concern, however, the required actuating voltage can be reduced by reducing the width of the beam and lateral spacing of the fixed electrodes [18]. Multiple beams can be used in conjunction with each other, similar to a comb-drive, to further reduce the driving voltage. A previous study by the authors had attempted to address this issue by supplying the side electrode voltage with a triboelectric generator [19]. It was demonstrated that triboelectricity has a synergistic relationship with electrostatic levitation and can be used to overcome the high voltage requirement by using a very simple and cheap triboelectric generator.

To use the MEMS actuator from [11] as a pressure switch, the beam is excited near its natural frequency through an AC voltage on the side electrodes (herein referred to as the side voltage). If the beam travels too close to the center electrode, dynamic pull-in is triggered and the switch is closed. When driven at or near the natural frequency, the oscillation amplitude is heavily dependent on squeeze film damping between the beam and substrate. A high ambient pressure creates significant squeeze film damping and the limits the oscillation amplitude. As the pressure decreases, the damping also decreases, which allows the oscillation amplitude to grow until it triggers dynamic pull-in and closes the switch. Therefore, for a given set of voltages on the side and center electrodes, there will be a threshold pressure below which the switch will automatically close. The pressure at which this happens is not only dependent on squeeze film damping, but also on the voltage applied to the center electrode (herein referred to as the bias voltage). This attribute allows the threshold pressure to be tuned by adjusting the bias voltage. This capability is not possible with conventional parallel-plate devices because the electrostatic force is so strong it can easily overcome the spring effect of the air cushion, making the dynamic pull-in voltage relatively insensitive to pressure.

A MEMS pressure switch that is tunable and reusable has great potential for many applications. This system provides a method for automatically triggering a response to a drop in pressure while maintaining the versatility and compact size of a MEMS device. The outline of this paper is as follows. In Section II a simple theoretical model of the system is derived. Section III describes the experimental setup and methods used to prove the feasibility of the pressure switching concept. Next, the results of the model and experiment are analyzed in Section IV. Lastly, a few concluding remarks are mentioned in Section V.

\section{Theoretical Model}

To create a simple model that can demonstrate the validity of the working principle, a lumped parameter model of a beam with electrostatic forcing is derived. This ordinary differential equation is based on Newtons 2 nd Law, $\vec{F}=m \vec{a}$, and is shown in Eq. (1),

$$
m \ddot{\hat{z}}=-c_{\text {air }}(\hat{z}) \dot{\hat{z}}-k \hat{z}-k_{\text {air }}(\hat{z}) \hat{z}+f_{e}\left(\hat{z}, V_{\text {side }}, V_{\text {bias }}\right)
$$

where $\hat{z}$ is the transverse displacement of the beam tip, $c_{\text {air }}(\hat{z})$ is the nonlinear damping function coming from the viscosity of the surrounding air, $k$ is the effective stiffness of the beam, $k_{\text {air }}(\hat{z})$ is the stiffness that comes the compressibility of the air, and $f_{e}\left(\hat{z}, V_{\text {side }}, V_{\text {bias }}\right)$ is the electrostatic force that depends on the applied voltages and beam displacement. For a cantilever beam, the effective mass $(m)$ and stiffness $(k)$ are well known and given by the relationships,

$$
m=0.646 \rho A_{c} L \quad k=\frac{8 E I}{L^{3}}
$$

where $A_{c}$ is the cross-sectional area, $\rho$ is the beam density, $L$ is the beam length, $E$ is the elastic modulus, and $I$ is the second moment of the area. The dimensions of the beam and electrodes, along with the relevant material properties are given in Table I.

The air terms, $c_{\text {air }}(\hat{z})$ and $k_{\text {air }}(\hat{z})$, are more difficult to calculate. For simplicity, these terms are estimated from wellestablished formulas to calculate the linear damping coefficient and linear air spring stiffness for squeezed air between two parallel plates [20]. Because the motion of the beam is 


\begin{tabular}{lcc}
\hline Parameter & Variable & Value \\
\hline Beam Length & $L$ & $500 \mu \mathrm{m}$ \\
Beam Width & $b_{3}$ & $20.5 \mu \mathrm{m}$ \\
Beam Thickness & $h_{3}$ & $2 \mu \mathrm{m}$ \\
Anchor Height & $d$ & $2 \mu \mathrm{m}$ \\
Electrode Side Lateral Distance & $g$ & $20.75 \mu \mathrm{m}$ \\
Side Electrode Width & $b_{1}$ & $32 \mu \mathrm{m}$ \\
Center Electrode Width & $b_{2}$ & $28 \mu \mathrm{m}$ \\
Electrode Thickness & $h_{1}$ & $0.5 \mu \mathrm{m}$ \\
Elastic Modulus & $\mathrm{E}$ & $166 \mathrm{GPa}$ \\
Density & $\rho$ & $2330 \mathrm{~kg} / \mathrm{m}^{3}$ \\
Poisson's Ratio & $\nu$ & 0.22 \\
\hline \multicolumn{3}{c}{ TABLE I } \\
\multicolumn{2}{c}{ BEAM PARAMETERS }
\end{tabular}

expected to be large, the gap between plates is modified to include the instantaneous deflection of the beam, leading to two nonlinear functions of $\hat{z}$, which become more significant when the beam gets close to the substrate. These terms are given as,

$$
\begin{gathered}
c_{\text {air }}(\hat{z})=\frac{64 \sigma P_{a} A}{\pi^{6} \Omega(\hat{z}+d)} \times \frac{1+\left(b_{3} / L\right)^{2}}{\left(1+\left(b_{3} / L\right)^{2}\right)^{2}+\sigma^{2} / \pi^{4}} \\
k_{\text {air }}(\hat{z})=\frac{64 \sigma^{2} P_{a} A}{\pi^{8}(\hat{z}+d)} \times \frac{1}{\left(1+\left(b_{3} / L\right)^{2}\right)^{2}+\sigma^{2} / \pi^{4}}
\end{gathered}
$$

where $P_{a}$ is the air pressure, $A$ is the overlapped area, $\Omega$ is the oscillation frequency in radians per second, and $\sigma$ is the squeeze number, which is given by,

$$
\sigma=\frac{12 A \Omega \mu_{e f f}}{P_{a}(\hat{z}+d)^{2}}
$$

where $\mu_{e f f}$ is the effective viscosity of the air.

The electrostatic force is also fairly complicated to model. This system has 3 electrodes in an unusual configuration with multiple applied voltages (assuming the two side electrodes in Figure 1 are treated as a single electrode). To calculate the electrostatic force, the potential energy of the beam is differentiated with respect to the transverse deflection, $\hat{z}$. The potential energy of an electrostatic system is given by $U=\frac{1}{2} C V^{2}$, where $C$ is the capacitance and $V$ is the applied voltage. Because the system has multiple electrodes, there are multiple capacitances and the potential energy is given by [11],

$$
U=\frac{1}{2}\left[\begin{array}{lll}
V_{\text {side }} & V_{\text {bias }} & V_{\text {beam }}
\end{array}\right]\left[\begin{array}{lll}
c_{11} & c_{12} & c_{13} \\
c_{21} & c_{22} & c_{23} \\
c_{31} & c_{32} & c_{33}
\end{array}\right]\left[\begin{array}{c}
V_{\text {side }} \\
V_{\text {bias }} \\
V_{\text {beam }}
\end{array}\right]
$$

where $c_{i j}$ are the mutual capacitances between each pair of electrodes, and $c_{i i}$ are the self capacitances. These capacitances are calculated numerically with a finite-element simulation in COMSOL. A 2D simulation of the switch using the cross section shown in Figure 1 is performed. The beam is meshed with very fine square elements and the surrounding air is meshed with triangular elements. The capacitances were calculated at gaps (between the beam and center electrode) from $0.1 \mu \mathrm{m}$ to $20 \mu \mathrm{m}$ at a step of $0.1 \mu \mathrm{m}$, which gives numerical profiles of the capacitance as a function of the beam z-direction displacement. A direct solver with a tolerance of $10^{-12}$ is used, which has matched very closely with previous experiments by the authors [11], [16], [17], [19].

To get the force in the z-direction, $V_{\text {beam }}$ is set to zero because the beam is the reference ground voltage level. Eq. (6) is expanded and the derivative is taken with respect to $\hat{z}$, yielding,

$$
\begin{aligned}
& f_{e}\left(\hat{z}, V_{\text {side }}, V_{\text {bias }}\right)= \\
& \quad \frac{1}{2}\left(V_{\text {side }}^{2} \frac{\partial c_{11}}{\partial \hat{z}}+2 V_{\text {side }} V_{\text {bias }} \frac{\partial c_{12}}{\partial \hat{z}}+V_{\text {bias }}^{2} \frac{\partial c_{22}}{\partial \hat{z}}\right)
\end{aligned}
$$

Analytical functions are fit to $\frac{\partial c_{11}}{\partial \tilde{z}}, \frac{\partial c_{12}}{\partial \tilde{z}}$, and $\frac{\partial c_{22}}{\partial \tilde{z}}$ to substitute in Eq. (1). 9th order polynomials are fit to $\frac{\partial c_{11}}{\partial \tilde{z}}$ and $\frac{\partial c_{12}}{\partial \tilde{z}}$. A high polynomial order is required to accurately match the numerical data over the the large range of displacement that the beam is predicted to experience. The last term, $\frac{\partial c_{22}}{\partial \hat{z}}$, is best fit with an inverse polynomial with a noninteger exponent. The analytical expression for electrostatic force is given in Eq. (8),

$$
\begin{gathered}
f_{e}\left(\hat{z}, V_{\text {side }}, V_{\text {bias }}\right)=\frac{1}{2} V_{\text {side }}^{2} \sum_{i=0}^{9} \alpha_{i} \hat{z}^{i}+ \\
V_{\text {side }} V_{\text {bias }} \sum_{i=0}^{9} \beta_{i} \hat{z}^{i}+\frac{1}{2} V_{\text {bias }}^{2} \frac{\gamma}{(\hat{z}+d)^{2.15}}
\end{gathered}
$$

where $\alpha_{i}, \beta_{i}$, and $\gamma$, are fitting coefficients.

Inserting Eq. (8) into Eq. (1) and nondimensionalizing with the relationships given in Eq. (9), yields the final nondimensional equation of motion,

$$
\begin{gathered}
z=\frac{\hat{z}}{h_{3}} \quad t=\frac{\hat{t}}{T} \quad \Omega=\hat{\Omega} T \quad T=\sqrt{\frac{\rho A_{c} L^{4}}{E I}} \\
\ddot{z}+c_{\text {air }}(z) \frac{T}{m} \dot{z}+\left(k+k_{\text {air }}(z)\right) \frac{T^{2}}{m} z= \\
V_{\text {side }}^{2} \sum_{i=0}^{9} A_{i} z^{i}+V_{\text {side }} V_{\text {bias }} \sum_{i=0}^{9} B_{i} z^{i}+V_{\text {bias }}^{2} \frac{G}{\left(z+\frac{d}{h_{3}}\right)^{2.15}}
\end{gathered}
$$

where $A_{i}, B_{i}$, and $G$ are defined as,

$$
A_{i}=\frac{\alpha_{i} h_{3}^{i-1} T^{2}}{2 m} \quad B_{i}=\frac{\beta_{i} h_{3}^{i-1} T^{2}}{m} \quad G=\frac{\gamma T^{2}}{2 m h_{3}^{3.15}}
$$

The highly nonlinear electrostatic and air effect terms make Eq. (11) almost impossible to solve analytically. Therefore, Eq. (11) is solved with numerical integration in MATLAB. The nonlinearity also introduces another complication to the switch. This system shows significant softening nonlinearities in the frequency response from both the side and bias voltage [11]. This makes driving the system at its natural frequency difficult because the natural frequency decreases as the oscillation amplitude increases. Therefore, instead of driving the system at a single frequency, the frequency of the AC voltage is swept backwards across the first natural frequency of the cantilever. This ensures that the resonant peak, which is very susceptible to changes in damping/pressure, is captured.

A constant pressure $\left(P_{a}\right)$ and bias voltage $\left(V_{\text {bias }}\right)$ are set and the side voltage frequency sweep is conducted. The side 


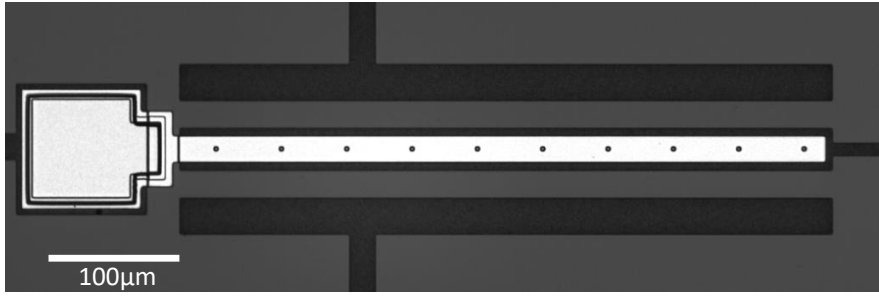

Fig. 2. Optical image of a fabricated beam.

voltage consists of a small $\mathrm{AC}$ voltage superimposed on top of a large DC voltage. The side DC voltage pushes the static position of the beam away from the substrate to give the beam more room to oscillate before hitting the center electrode. A large oscillation is desired to maximize the air spring and nonlinear squeeze film damping effects, which must influence the dynamics of the beam for the switch to work.

To see if the switch closes near the resonant peak at the applied bias voltage and ambient pressure, the time response of the beam is monitored for stability. If the solution becomes unstable (i.e. pull-in occurs), the beam response will begin to move rapidly in the negative direction (downward, towards the center electrode) and the solver will stop. The bias voltage is updated and the process is repeated until the threshold bias voltage to trigger dynamic pull-in is found for the given pressure level. The purpose is to extract a trend between switching voltage and ambient pressure, showing the dynamic pull-in voltage is a function of pressure.

\section{EXPERIMENTAL Methods}

The same technique applied to the model to extract the relationship between dynamic pull-in voltage and ambient pressure is repeated in an experiment to verify the validity of the model results. The switch is fabricated with PolyMUMPs [21] to the dimensions in Table I. An image of a fabricated beam is shown in Figure 2. The beam is placed in a vacuum chamber and the tip velocity is measured with a Polytec MSA-500 laser vibrometer. $170 V_{\text {side } D C}$ is applied to the side electrodes and gives an initial deflection of approximately $11 \mu \mathrm{m}$. With the anchor height of $2 \mu \mathrm{m}$, this gives an initial gap of around $13 \mu \mathrm{m}$. The large DC voltage on the side electrodes pushed the beam upward and increases the initial gap, which gives the beam room to oscillate. A large oscillation amplitude is desired to maximize the effect of the air spring and nonlinear damping and increase the influence of pressure on the dynamic pull-in voltage. Once the pressure in the vacuum chamber stabilizes, a bias voltage from $6-12 \mathrm{~V}$ is applied to the center electrode. When the bias voltage is turned on, the beam will be pulled back down slightly. Because the side voltage is much larger than the bias voltage, it only pulls the beam down around $1-2 \mu \mathrm{m}$ at most, leading to an actual initial gap of around $11-12 \mu \mathrm{m} .1 V_{\text {side } A C}$ is superimposed on the side electrode DC voltage and the frequency is swept downward from $13 \mathrm{kHz}$ to $8 \mathrm{kHz}$ across the first natural frequency of the beam $\left(10.5 \mathrm{kHz}\right.$ at $\left.170 V_{D C}\right)$. A schematic of the experimental setup is shown in Figure 3.

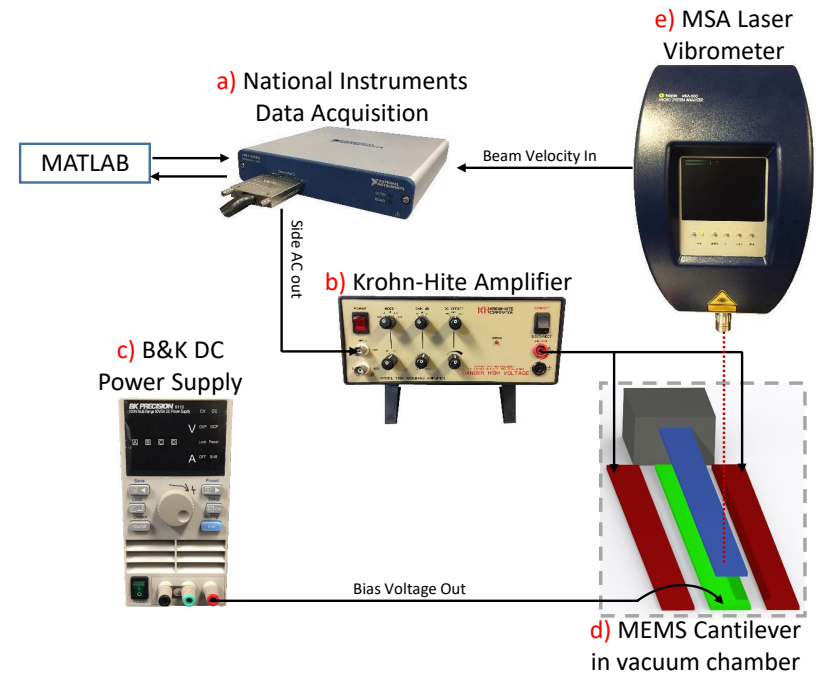

Fig. 3. Set up of the experiment with the specific equipment used in the experiment.

The bias voltage is increased incrementally and the experiment is repeated until the beam experiences dynamic pull-in near the resonant peak. The minimum voltage needed to induce pull-in at the given pressure is recorded, and this process is repeated many times for vacuum pressures between 0.7 and 1.6 Torr.

\section{Results AND Discussion}

Prior to the experiment, the profile of the beam with no applied voltage is measured with a Wyko NT1100 optical profiler (Figure 4). There is a notable curling of the beam from residual stress and the tip is curled up almost $2.5 \mu \mathrm{m}$. This gives an initial gap at the tip of around $4.5 \mu \mathrm{m}$ (as opposed to $2 \mu \mathrm{m}$ at the anchor). This is very important for the model because it has a noticeable effect on the dynamic pull-in voltage. The curled tip causes the actual gap between the beam and center electrode to be slightly larger than it would be for a perfectly flat beam, which is what the model assumes. To account for this, the actual gap along the length of the beam for the fabricated switch is measured and averaged. The anchor height of the beam in the model $(d)$ is adjusted to this average value, which is found to be approximately $2.5 \mu \mathrm{m}$. A similar technique was employed in [11] and was shown to give very good agreement with experimental results.

After the initial profile of the beam is measured, the dynamic test is conducted. Figure 5 shows the tip velocity time data at 1.39 Torr and a bias voltage of (a) $9.9 \mathrm{~V}$ and (b) $10 \mathrm{~V}$. As the AC frequency approaches the natural frequency $(10.5 \mathrm{kHz})$, the oscillation amplitude increases significantly before the switch experiences hysteresis and the amplitude drops suddenly after the natural frequency is passed. The experiment confirms the highly nonlinear behavior that comes from the electrostatic force. Because the frequency is swept downward, this is a softening nonlinearity, which agrees with previous experiments by the authors [11], [16], [17].

For the $9.9 \mathrm{~V}$ bias case, when hysteresis occurs the beam remains stable and falls to a low amplitude stable oscillation 


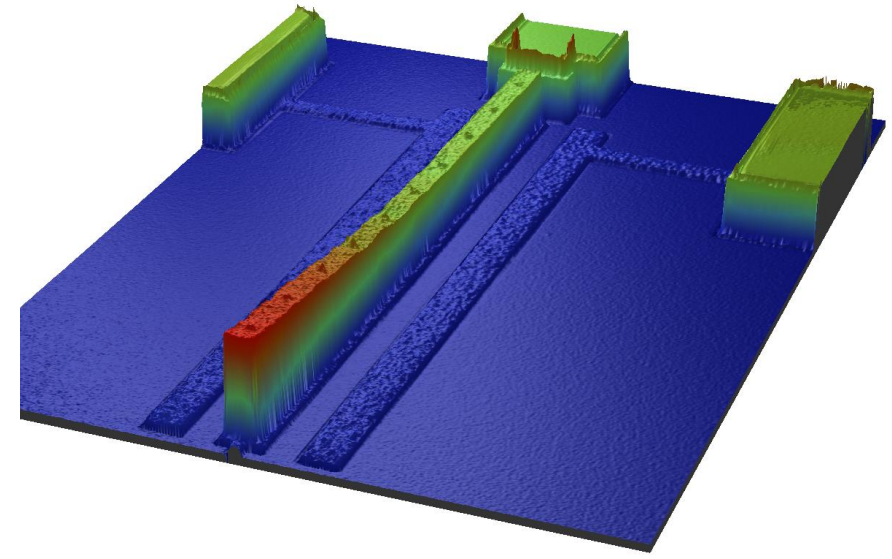

Fig. 4. Beam profile with no applied voltage showing the curling of the tip from residual stress during the fabrication process.
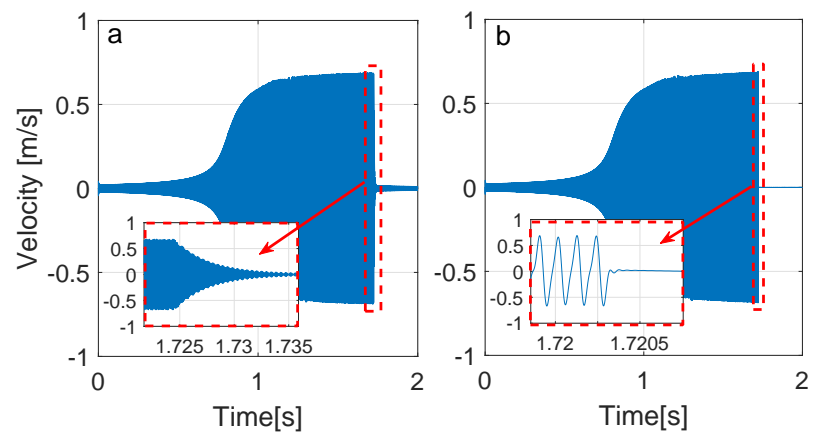

Fig. 5. Beam tip velocity vs time at at $170 V_{\text {side }_{D C}}, 1 V_{\text {side }_{A C}}, 1.39$ Torr and a) $9.9 V_{\text {bias }}$ and b) $10 V_{\text {bias }}$. The frequency is swept downwards from $13 \mathrm{kHz}$ to $8 \mathrm{kHz}$. The beam does not experience pull-in at $9.9 \mathrm{~V}$, but does at $10 \mathrm{~V}$. The inlet of both figures shows how the response behaves after the natural frequency has been passed.

as seen in the inlet of Figure 5 (a), which shows a zoomed in portion of the plot at around 1.8 seconds. At this voltage, the switch remains open and is not triggered. However, when the voltage is increased by just $0.1 \mathrm{~V}$ to $10 \mathrm{~V}$, instead of remaining stable and falling to a low amplitude oscillation, the beam becomes unstable and triggers dynamic pull-in. This is highlighted in the inlet of Figure 5 (b), which shows the tip velocity suddenly falling to zero. The oscillation disappears because the beam is stuck to the substrate and can not move anymore. When dynamic pull-in occurs, the switch is closed. Therefore, to trigger the switch when the pressure drops below 1.39 Torr, $10 \mathrm{~V}$ must be applied to the center electrode.

To gain more insight into the beam dynamics, a zoomed in portion of the data in Figure 5 (b) at approximately $1.7 \mathrm{~s}$ is shown in Figure 6. Also shown in Figure 6 is the dynamic displacement, which is calculated by integrating the velocity signal in the frequency domain. To avoid drift, low frequency noise under $100 \mathrm{~Hz}$ is set to zero. The dynamic displacement data is included because it is much easier to interpret than the velocity data.

As can be seen in the velocity data from Figure 6, there is noticeable nonlinear behavior when the beam crosses the zero
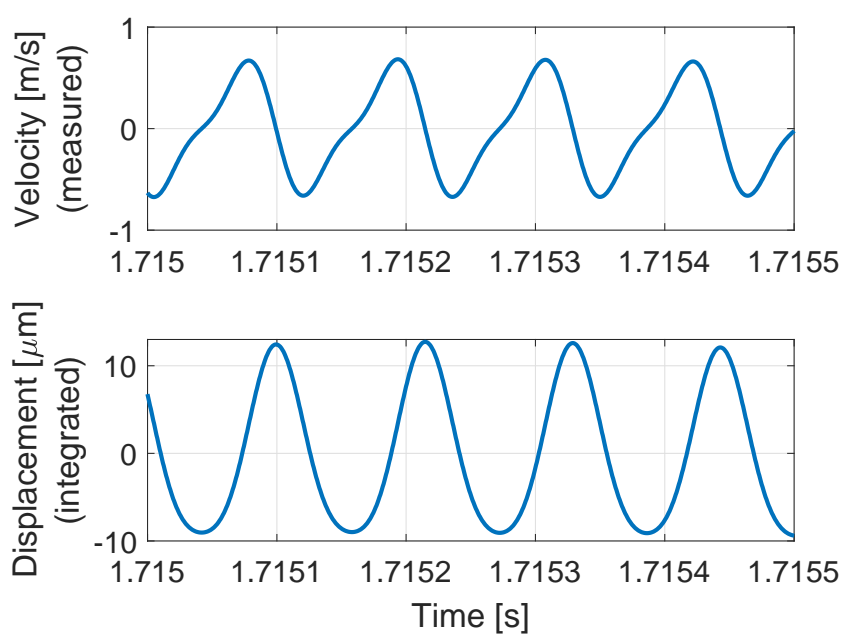

Fig. 6. Zoomed in velocity data from Figure 5 (b) at approximately $1.7 \mathrm{~s}$ with estimated dynamic displacement showing a peak-to-peak oscillation of $20 \mu \mathrm{m}$.

velocity axis from the negative direction. In this case, negative velocity is when the beam is traveling downward, towards the substrate. Therefore, this nonlinear behavior occurs when the beam is at its lower extreme and the gap is small. The peakto-peak dynamic displacement is approximately $22 \mu \mathrm{m}$, which is very large considering the anchor height of the beam is only $2 \mu \mathrm{m}$. The large oscillation is only possible because the high DC voltage on the side electrodes pushes the static position away from the substrate and gives the beam more room to oscillate. The displacement also shows this nonlinear effect when the beam is close to the substrate where it appears to slow down.

While at first glance the nonlinear response may seem to be a result of nonlinear damping that becomes significant and slows down the beam velocity when the gap is small, the model shows this is actually not the case. The cause of this behavior is the bias voltage and its effect on the linear stiffness of the beam. Similar to a parallel-plate system, the bias voltage decreases the linear stiffness, which makes the system "softer". The authors have previously demonstrated in [16] that electrostatic levitation from the side voltage increases the linear stiffness. When the beam is close to the center electrode, the bias voltage dominates and the beam is softer than when the gap is large (where the side voltage is dominant). This causes the frequency to be slightly lower at small gaps than at large gaps, which creates the time signal in Figure 6.

To illustrate this point, high amplitude motion is simulated with the model at $170 V_{\text {side }_{D C}}, 1 V_{\text {side }_{A C}}$, and a pressure of 1.4 Torr. Two cases are examined; no bias voltage (Figure 7), and $10 V_{\text {bias }}$ (Figure 8). In Figure 7, when there is no bias voltage, the time response looks relatively linear. In this case, the total displacement of the beam is $22 \mu \mathrm{m}$ peak-topeak and is still heavily influenced by the air because the motion is large. It is important to note that this is total displacement and not dynamic displacement like in Figure 6. If the nonlinear time signal is a result of nonlinear damping 

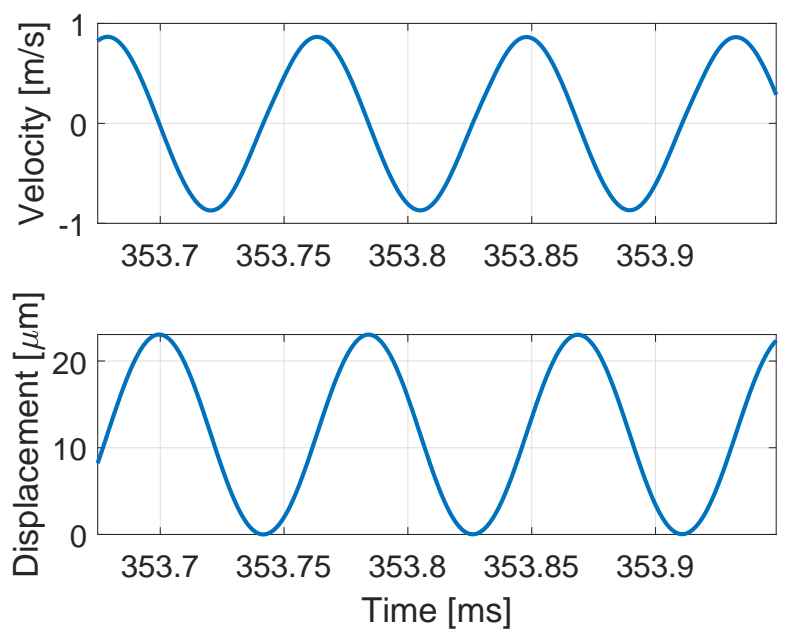

Fig. 7. Velocity and total displacement results from the model at $170 V_{\text {side }_{D C}}, 1 V_{\text {side }_{A C}}, 0 V_{\text {bias }}$ and 1.4 Torr.
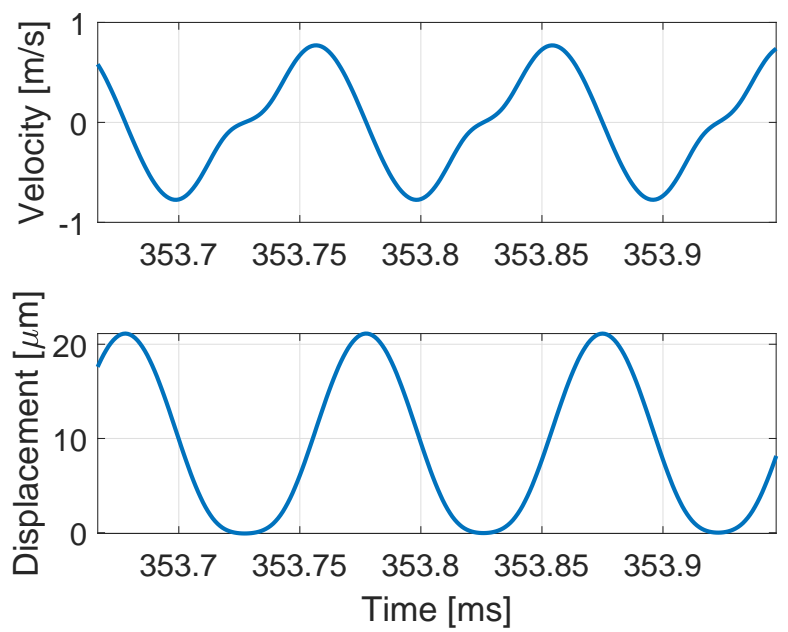

Fig. 8. Velocity and total displacement results from the model at $170 V_{\text {side }_{D C}}, 1 V_{\text {side }_{A C}}, 10 V_{\text {bias }}$ and 1.4 Torr.

and/or the air spring effect, it should show up in Figure 7, however, it does not. If the bias voltage is turned to $10 \mathrm{~V}$ and the simulation is repeated, the nonlinearity appears in the time signal while the peak-to-peak amplitude remains relatively the same. Therefore, this behavior is not from the surrounding air, but from the bias voltage.

Although the bias voltage is affecting the motion of the beam even when it remains stable, this is not problematic for the pressure switch application. Unlike a pressure sensor, there is no continuous output signal of a pressure switch and it only has two states, open and closed. While the time behavior in Figures 6-8 is interesting and unusual, it does not negatively impact the performance of the switch. To demonstrate the working principle of the pressure switch, the experiment is repeated for many pressure levels between 0.7 and 1.6 Torr. The relationship between pressure and threshold pull-in voltage for the model and experiment is shown in

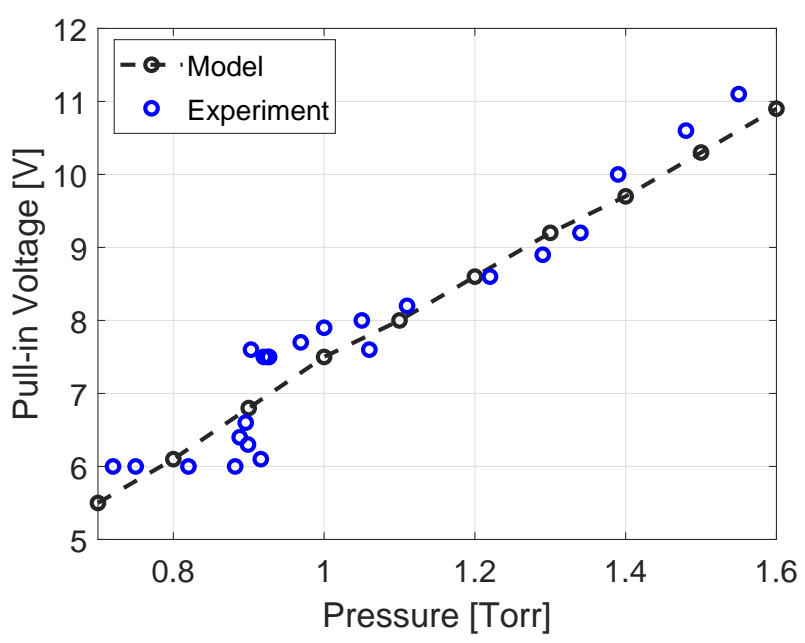

Fig. 9. Pressure vs pull-in voltage at various pressures for both the model and experimental data. The black dashed line shows the results for the model with an effective anchor height of $2.5 \mu \mathrm{m}$ to account for curling of the beam tip from residual stress during fabrication.

Figure 9.

Similar to the data in Figure 5, the points in Figure 9 are recorded by setting a constant vacuum pressure and sweeping the $\mathrm{AC}$ voltage on the side electrodes across the first natural frequency. The threshold bias voltage to initiate dynamic pullin when the frequency sweep hits the resonant peak is recorded for each pressure. The model and experiment agree with each other and show an upward trend in pull-in voltage with increasing pressure. This proves the pressure switch concept is valid. High ambient pressure limits the oscillation amplitude and requires a higher bias voltage to initiate dynamic pull-in and trigger the switch. For example, to trigger the switch when the pressure drops below 1 Torr, a bias voltage of $8 \mathrm{~V}$ should be applied to the center electrode. But if the bias voltage is set to $10 \mathrm{~V}$, the switch will trigger at 1.39 Torr instead. A great advantage of this system is that after the switch has been triggered, the device can be reset to its initial state by removing the bias voltage. This allows the electric field from the side voltage to pull the beam out of its pulled-in position and the switch can be reused. In the experiment, the same device was used for all the data points in Figure 9, which required the beam to be released over 20 times. This had no noticeable effect on the performance of the device for subsequent tests.

As the pressure drops under 0.9 Torr, the pull-in voltage drops suddenly to around $6 \mathrm{~V}$ and stays constant. It is expected that the pressure switching concept will stop working at very low pressures because the influence of the air will become too small to have a noticeable effect on the dynamic pull-in voltage. For very low pressures, the damping of the beam will come mostly from other damping sources such as thermoelastic effects and anchor loss, while the air spring effect will be negligible. The minimum pressure at which this occurs is primarily a function of the switch geometry, however, in this case the pressure switching mechanism ceases to work below 0.9 Torr.

It is also expected that at very high pressures the influence of 
the air is very large and requires a high bias voltage to trigger dynamic pull-in. This is not as problematic as the low-pressure limitation of the switch because it can be addressed in several ways. The most straightforward way is to increase the driving side voltage. This will increase the oscillation amplitude and decrease the threshold pull-in voltage. However, this may also run into similar issues where a very high driving side voltage is required to counteract the large viscous damping and air spring forces if the pressure is well above atmospheric pressure. Another way to address this problem is to alter the switch geometry such that the natural frequency is lower. The air spring effect [Eq. (4)] is proportional to the square of the driving frequency, meaning it is more significant at high frequencies than at lower frequencies. The air spring has a considerable effect on the beam amplitude near the resonant peak and by decreasing its influence with a lower operating frequency, the dynamic pull-in voltage will be smaller.

At 0.9 Torr, the experiment shows some unusual behavior. There is a sudden drop in pull-in voltages from about $7.5 \mathrm{~V}$ to $6 \mathrm{~V}$. This drop is not seen in the model and therefore must be coming from something that was excluded from the modeling process in Section II. The most likely candidate is the motion of the surrounding air. The air has its own behavior that is governed by the Navier-Stokes equation. In the model of the switch, the full Navier-Stokes equation was not considered. Instead, the effect of the air was characterized by an air spring and nonlinear damping effect. Other factors such as added mass, and resonances of the air were ignored for simplicity.

The motion of the air would be very difficult to model accurately because of the large oscillation of the beam in very close proximity to the substrate. This would require coupling the nonlinear Navier-Stokes equation with the Euler-Bernoulli beam equation to get a full model of the entire system. However, the purpose of this study is simply to demonstrate the feasibility of the MEMS pressure switch and a much simpler model is used. Despite the discrepancy between the model and the experiment below 0.9 Torr, the results clearly show a trend between pressure and threshold bias voltage for pull-in, and therefore the pressure sensing concept is feasible.

The design is also compatible with almost any other commercially available MEMS switch as side electrodes on either side of the switch can be easily added to the fabrication layouts. Traditional MEMS switches just utilize the center electrode to pull the beam (source) down into contact with a drain electrode. This design uses the same switching mechanism, except it operates in a dynamic mode instead of a static mode. This means that most methods of increasing reliability, life-span, insertion loss, and isolation should also work for this switch.

\section{CONCLUSION}

In this study, a tunable MEMS pressure switch is introduced. The switch exploits the significant coupling between beam and fluid dynamics that occur with large beam oscillations from electrostatic levitation. It is theoretically and experimentally demonstrated that the threshold bias voltage to induce dynamic pull-in is a function of the ambient pressure and can be used to control the triggering pressure of the switch. The device requires no computational overhead to trigger the switch as the system operates entirely through mechanical and electrical phenomena that happen automatically. The bias voltage allows the triggering pressure to be adjusted so the same switch can be tuned to different applications and specifications. This design is very useful for systems that benefit from the advantages of MEMS technology, but only need to trigger a response when the pressure drops below a threshold value.

\section{RefERENCES}

[1] W. P. Eaton and J. H. Smith, "Micromachined pressure sensors: review and recent developments," Smart Materials and Structures, vol. 6, no. 5, p. $530,1997$.

[2] A. Shirinov and W. Schomburg, "Pressure sensor from a pvdf film," Sensors and Actuators A: Physical, vol. 142, no. 1, pp. 48-55, 2008.

[3] H. Takahashi, N. M. Dung, K. Matsumoto, and I. Shimoyama, "Differential pressure sensor using a piezoresistive cantilever," Journal of micromechanics and microengineering, vol. 22, no. 5, p. 055015, 2012.

[4] M. H. Hasan, F. M. Alsaleem, and H. M. Ouakad, "Novel threshold pressure sensors based on nonlinear dynamics of mems resonators," Journal of Micromechanics and Microengineering, vol. 28, no. 6, p. 065007, 2018

[5] N. Boechler, G. Theocharis, and C. Daraio, "Bifurcation-based acoustic switching and rectification," Nature materials, vol. 10, no. 9, p. 665, 2011.

[6] V. Kumar, J. W. Boley, Y. Yang, H. Ekowaluyo, J. K. Miller, G. T. C. Chiu, and J. F. Rhoads, "Bifurcation-based mass sensing using piezoelectrically-actuated microcantilevers," Applied Physics Letters, vol. 98 , no. 15 , p. $153510,2011$.

[7] V.-N. Nguyen, S. Baguet, C.-H. Lamarque, and R. Dufour, "Bifurcationbased micro-/nanoelectromechanical mass detection," Nonlinear Dynamics, vol. 79, no. 1, pp. 647-662, 2015.

[8] R. Harne and K. Wang, "A bifurcation-based coupled linear-bistable system for microscale mass sensing," Journal of Sound and Vibration, vol. 333, no. 8, pp. 2241-2252, 2014.

[9] R. A. Dias, E. Cretu, R. Wolffenbuttel, and L. A. Rocha, "Pull-inbased $\mu$ g-resolution accelerometer: Characterization and noise analysis," Sensors and Actuators A: Physical, vol. 172, no. 1, pp. 47-53, 2011.

[10] M. Khater, M. Al-Ghamdi, S. Park, K. Stewart, E. Abdel-Rahman, A. Penlidis, A. Nayfeh, A. Abdel-Aziz, and M. Basha, "Binary mems gas sensors," Journal of Micromechanics and Microengineering, vol. 24, no. 6, p. 065007, 2014.

[11] M. Pallay, R. N. Miles, and S. Towfighian, "Merging parallel-plate and levitation actuators to enable linearity and tunability in electrostatic mems," Journal of Applied Physics, vol. 126, no. 1, p. 014501, 2019.

[12] S. He and R. Ben Mrad, "Large-stroke microelectrostatic actuators for vertical translation of micromirrors used in adaptive optics," IEEE Transactions on Industrial Electronics, vol. 52, no. 4, pp. 974-983, 2005.

[13] C. Fan and S. He, "A Two-Row Interdigitating-Finger RepulsiveTorque Electrostatic Actuator and Its Application to Micromirror Vector Display," Journal of Microelectromechanical Systems, vol. 24, DOI 10.1109/JMEMS.2015.2467389, no. 6, pp. 2049-2061, 2015.

[14] K. B. Lee and Y. H. Cho, "Laterally driven electrostatic repulsive-force microactuators using asymmetric field distribution," Journal of Microelectromechanical Systems, vol. 10, DOI 10.1109/84.911101, no. 1, pp. 128-136, 2001

[15] M. Pallay and S. Towfighian, "A reliable MEMS switch using electrostatic levitation," Applied Physics Letters, vol. 113, DOI 10.1063/1.5053090, no. 21, p. 213102, 2018.

[16] M. Pallay, M. Daeichin, and S. Towfighian, "Dynamic Behavior of an Electrostatic MEMS Resonator with Repulsive Actuation," Nonlinear Dynamics, vol. 89, DOI 10.1007/s11071-017-3532-z, no. 2, pp. 15251538, 2017.

[17] M. Pallay and S. Towfighian, "A parametric electrostatic resonator using repulsive force," Sensors and Actuators A: Physical, vol. 277, DOI 10.1016/j.sna.2018.04.001, pp. 134 - 141, 2018.

[18] S. Towfighian, S. He, and R. Ben Mrad, "A low voltage electrostatic micro actuator for large out-of-plane displacement," in ASME 2014 International Design Engineering Technical Conferences and Computers and Information in Engineering Conference. American Society of Mechanical Engineers Digital Collection, 2014. 
[19] M. Pallay, A. I. Ibrahim, R. N. Miles, and S. Towfighian, "Pairing electrostatic levitation with triboelectric transduction for high-performance self-powered mems sensors and actuators," Applied Physics Letters, vol. 115 , no. 13 , p. $133503,2019$.

[20] J. J. Blech, "On isothermal squeeze films," Journal of lubrication technology, vol. 105, no. 4, pp. 615-620, 1983.

[21] A. Cowen, B. Hardy, R. Mahadevan, and S. Wilcenski, "PolyMUMPs Design Handbook a MUMPsÂ® process,” 2011.

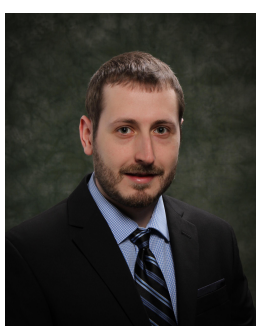

$\mathbf{M}$ ark Pallay was born in Ambler Pennsylvania. He received his B.S. and M.S. degrees in mechanical engineering at Binghamton University, Binghamton, New York in 2014 and 2016 respectively.

$\mathrm{He}$ is currently a $\mathrm{PhD}$ candidate and research assistant in the MEMS and Energy Harvesting $\mathrm{Lab}$ at Binghamton University. His research interests include MEMS sensors, vibrations, and nonlinear dynamics.

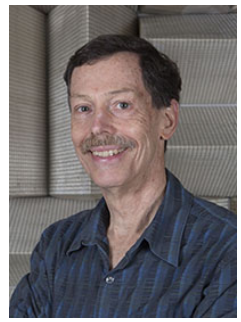

$\mathbf{R}$ onald N. Miles received his B.S. degree in electrical engineering from the University of California at Berkeley, Berkeley, California, and his M.S. and Ph.D. degrees in mechanical engineering from the University of Washington. He has been with the Department of Mechanical Engineering, SUNY Binghamton, Binghamton, NY, USA, since 1989. He has served as the Director of Graduate Studies, the Director of Undergraduate Studies, Associate Chair, Profor Research. fessor, Distinguished Professor, Associate Dean

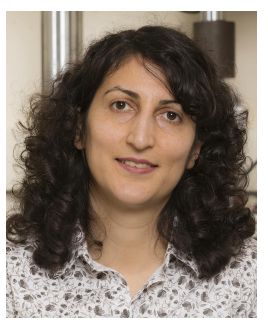

S hahrzad Towfighian received her BS degree in mechanical engineering in 2001 from Amirkabir University of Technology, Tehran, Iran. She earned her MS and PhD degrees from Ryerson University and the University of Waterloo, Canada in 2006 and 2011, respectively.

She worked as a Mechanical Design Engineer in hydro turbine industry in Iran for three years before conducting her graduate studies. She joined the Mechanical Engineering Department rently an Associate Professor. at Binghamton University in 2013 and is cur-

Prof. Towfighian's main areas of research are on MEMS and mechanical vibration energy harvesting. Her work has been published in more than 60 journal and international conference papers. She has been the recipient of several awards from the National Science Foundation and the National Institute of Health. Prof. Towfighian is a professional member of ASME and IEEE organizations. 